\title{
Simulating Progressive Failure of CFRP Materials with Fiber Waviness and Resin Zones
}

\author{
Zhihua Ning, Chunxiu Xu, and Guanliang Huo
}

\begin{abstract}
The progressive failure of carbon fiber reinforced polymer (CFRP) laminates with out-of-plane fiber waviness and resin zones is simulated in the present paper. A finite element model, which includes multiple damage modes, is developed for the static compression failure analysis of the laminates. Three-dimensional Hashin failure criteria are applied for damage onset and abrupt degradation was adopted for material degeneration. Both the case of a single resin zone and two resin zones are discussed. Different damage modes are investigated up to final failure. The results show that, fiber fracture is the dominant damage mechanism in both the case of a single resin-zone and two resin-zones, but inter-ply delamination also plays an essential role on the failure of the laminate in the case of two resin-zones. The predicted patterns of damage indicate that kink bands occur at the junction region of the two resin-zones, with severe fiber fracture and delamination damage. The effects of defect configuration on compressive strength are also quantitatively assessed.
\end{abstract}

Index Terms-Compressive strength, fiber waviness, progressive damage, resin-zone.

\section{INTRODUCTION}

Fiber waviness is one type of the common manufacturing defects particularly encountered during the filament-winding process. Various causes of the occurrences of fiber waviness may include the volume contraction due to chemical or thermal shrinkage [1], the mismatched size between components and mold [2], or consolidation proving excess length of material [3] are the common factors in terms of the waviness. It also could be summarized into non-uniform consolidation pressure, interactions with other layers or ply drop-offs [4].

The mechanical performance of the laminates is affected by the waviness, such as static strength and stiffness, and a large volume of work has been conducted on this topic [1-9]. It is note-worthy that fiber waviness in CFRP composite materials often companies resin zone or resin pocket. However, there are limited references in the literature considering the effect of the resin zone. Reference [10] investigated the influence of the out-of-plane waviness and resin zone on the static strength and fatigue life experimentally and numerically, in which the CFRP was modeled as transversely isotropic material without plies. Thus the FE model proposed in [10] is not a progressive

Manuscript received July 10, 2018; revised December 23, 2018. This work was supported by the National Natural Science Foundation of China under Grant No.11302083.

Z. Ning, C. $\mathrm{Xu}$, and G. Huo are with the School of Mechanics and Construction Engineering, MOE Key Lab of Disaster Forecast and Control in Engineering, Jinan University, Guangzhou, 510632, China (e-mail: ning_zhihua@163.com,864417966@qq.com,huog10515@163.com). damage model, without the ability to capture the damage mode sequence and to confirm the leading damage mechanism in the laminate.

In the present work, a FE model with multiple damage modes was developed to investigate the progressive failure of CFRP laminates with out-of-plane waviness accompanied by resin zones under axial compression. The FE results successfully illustrated the initiation of multiple interacting damage modes at the waviness location and the progressive failure. The influence of waviness geometry on the compressive strength reduction was also evaluated quantitatively.

\section{FINITE ELEMENT MODELLING}

\section{A. Model Description}

The out-of-plane waviness geometry is usually characterized by the wave severity, i.e., the ratio of wave amplitude over wavelength $\mathrm{A} / \mathrm{L}$, or the maximum misalignment angle $\theta_{\max }$ (see Fig. 1). In most of the literature involving waviness, sine or cosine waves are usually considered for describing and quantifying the waviness [5]-[9], typically in analytical models. Reference [4] presented a photo of a sever fiber waviness with resin pocket in a composite structure, in which the profile of the waviness looks like a bell-curve(see in Fig. 2) and was characterized by means of a Gaussian function.

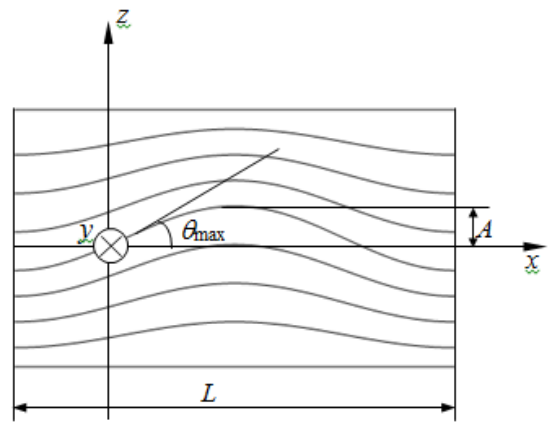

Fig.1. Schematic of waviness.

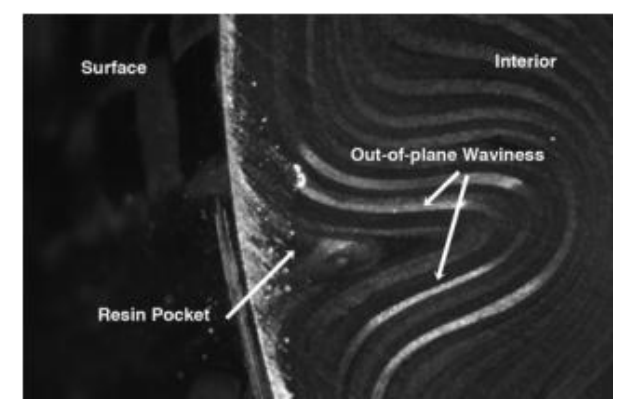

Fig. 2. Example of a severe fiber waviness profile in [4]. 
In experimental investigation, a means of artificially introducing and reproducing the waviness has to be considered. Thus a convenient way was proposed in [10] as follows: out-of-plane fiber waviness is reproduced by inserting polymer rods transverse to the fiber direction among the layers in a unidirectional laminate. In order to correlate the FE analysis with the experimental results in [10], a unidirectional (UD) CFRP laminate with local waviness and resin-zone similar to [10] is considered in the present work. The geometry of the composite laminate is shown in Fig.3. The laminate consists of twelve UD layers with a constant thickness of $1.8 \mathrm{~mm}$, the length and width of $20 \mathrm{~mm}$ and $6 \mathrm{~mm}$, respectively. The boundary between resin-zone and CFRP is approximated by spline curves and the inserted polymer rod is considered of circular cross-section with a diameter of $0.5 \mathrm{~mm}$ The width of the resin-zone is $2.4 \mathrm{~mm}$.

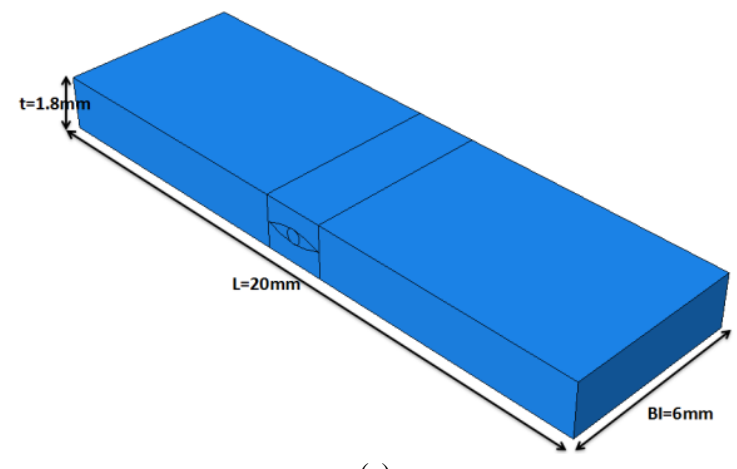

(a)

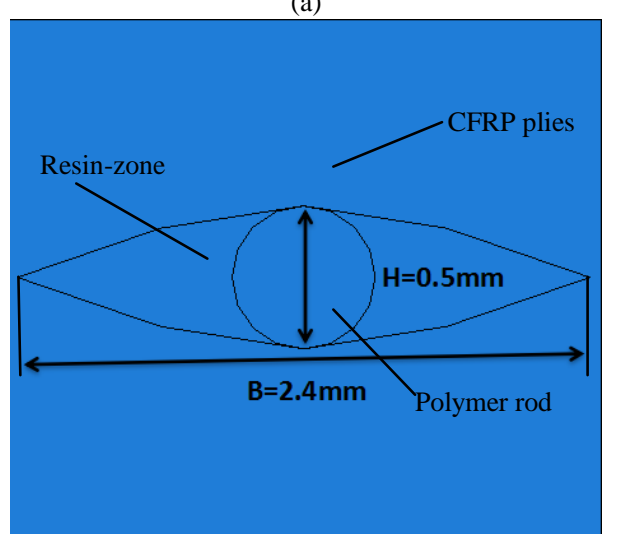

(b)

Fig. 3. Geometry of the composite laminate with a single resin-zone:(a) dimension of the laminate and (b) geometry of local waviness with resin zone.

The mechanical properties of the composite ply are shown in Table I. The Young's moduli of the polymer rod and the resin zone are $E_{\text {rods }}=1 \mathrm{GPa}, E_{\text {resin }}=3.4 \mathrm{GPa}$ respectively, and a Poisson's ratio $v=0.3$ is assumed.

TABLE I: MATERIAL PROPERTIES OF COMPOSITE

\begin{tabular}{cccccc}
\hline \hline$E_{11}$ & $E_{22}=E_{3}$ & $\mathrm{v}_{12}=\mathrm{v}_{13}$ & $\mathrm{v}_{23}$ & $G_{12}=G_{13}$ & $G_{23}$ \\
\hline $128 \mathrm{GPa}$ & $8.7 \mathrm{GPa}$ & 0.32 & 0.3 & 4.0 & 4.0 \\
\hline$X_{T}$ & $X_{C}$ & $Y_{T}=Z_{T}$ & $Y_{C}=Z_{C}$ & $S_{12}=S_{13}$ & $S_{23}$ \\
\hline $\begin{array}{c}2.093 \mathrm{GP} \\
\mathrm{a}\end{array}$ & $\begin{array}{c}0.87 \mathrm{GP} \\
\mathrm{a}\end{array}$ & $0.05 \mathrm{GPa}$ & $\begin{array}{c}0.198 \mathrm{GP} \\
\mathrm{a}\end{array}$ & $\begin{array}{c}0.104 \mathrm{GP} \\
\mathrm{a}\end{array}$ & $\begin{array}{c}0.086 \mathrm{GP} \\
\mathrm{a}\end{array}$ \\
\hline \hline
\end{tabular}

The FE model of the laminate shown in Fig. 4 was built up in ABAQUS/Standard. The layers are meshed using a sweep mesh with a defined sweep path and a refined mesh in the resin zone. The whole model is discretized with C3D8R continuum solid element of an average element size of $0.15 \mathrm{~mm}$ in the composite layers and $0.05 \mathrm{~mm}$ in the resin zone. For checking mesh convergence, uniform mesh refinement (2/3 element size in the composite layers) from 205219 to 435800 elements has shown an increase of $0.4 \%$ for the stresses at location $\mathrm{A}$ in the composite plies, while the stress level keep constant at location B in the resin zone. The load case considered for the FE model is compression displacement applied at the right end of the model in the longitudinal direction by the use of MPCs. Displacement in the $y$ direction at the ends of the model are suppressed. The displacement in the $x$ direction at the left end is also suppressed.

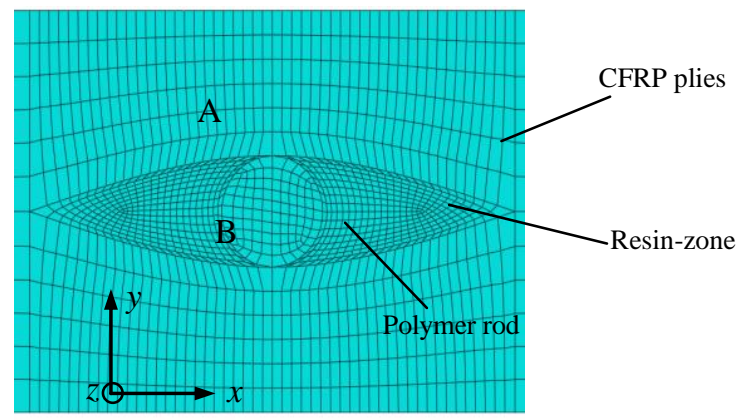

Fig. 4. FE mesh at waviness location.

\section{B. Multi-damage Initiation Criteria}

For the failure of a composite laminate with fiber waviness in compression, both in-plane failure and inter-ply delamination should be taken into account. Basically, there are three different in-plane failure modes in CFRP composite: fiber breakage, matrix cracking and fiber-matrix shearing. In the following analysis, three-dimensional Hashin failure criteria would be adopted to simulate the onset of multiple damages in the laminate, which can be expressed as follows.

Tensile fiber mode (for $\sigma_{11}>0$ )

$$
e_{f}=\left(\frac{\sigma_{11}}{X_{T}}\right)^{2}+\left(\frac{\sigma_{12}}{S_{12}}\right)^{2}+\left(\frac{\sigma_{13}}{S_{13}}\right)^{2} \geq 1
$$

Compressive fiber mode (for $\sigma_{11}<0$ )

$$
e_{f}=\left(\frac{\sigma_{11}}{X_{C}}\right)^{2} \geq 1
$$

Tensile matrix mode (for $\sigma_{22}>0$ )

$$
e_{m}=\left(\frac{\sigma_{22}}{Y_{T}}\right)^{2}+\left(\frac{\sigma_{12}}{S_{12}}\right)^{2}+\left(\frac{\sigma_{23}}{S_{23}}\right)^{2} \geq 1
$$

Compressive matrix mode (for $\sigma_{22}<0$ )

$$
e_{m}=\left(\frac{\sigma_{22}}{Y_{C}}\right)^{2}+\left(\frac{\sigma_{12}}{S_{12}}\right)^{2}+\left(\frac{\sigma_{23}}{S_{23}}\right)^{2} \geq 1
$$

Fiber-matrix shearing mode (for $\sigma_{11}<0$ )

$$
e_{s}=\left(\frac{\sigma_{11}}{X_{C}}\right)^{2}+\left(\frac{\sigma_{12}}{S_{12}}\right)^{2}+\left(\frac{\sigma_{13}}{S_{13}}\right)^{2} \geq 1
$$

Delamination in tension (for $\sigma_{33}>0$ ) 


$$
e_{d}=\left(\frac{\sigma_{33}}{Z_{T}}\right)^{2}+\left(\frac{\sigma_{13}}{S_{13}}\right)^{2}+\left(\frac{\sigma_{23}}{S_{23}}\right)^{2} \geq 1
$$

Delamination in compression (for $\sigma_{33}<0$ )

$$
e_{d}=\left(\frac{\sigma_{33}}{Z_{C}}\right)^{2}+\left(\frac{\sigma_{13}}{S_{13}}\right)^{2}+\left(\frac{\sigma_{23}}{S_{23}}\right)^{2} \geq 1
$$

In (1)-(7), $e_{f}, e_{m}, e_{s}, e_{d}$ are the damage threshold parameters of fiber breakage, matrix cracking, fiber-matrix shearing and delamination, respectively. $\sigma_{i j}(i, j=1,2,3)$ is the stress component referred to the principal material axes. $X_{T}$ and $X_{C}$ represent longitudinal tensile and compressive strengths; $Y_{T}$ and $Y_{C}$ are transverse tensile and compressive strengths; $S_{12}, S_{13}, S_{23}$ denote shear strengths of plane 1-2, 1-3 and $2-3$, respectively.

TABLE II: MATERIAL DEGRADATION

\begin{tabular}{|c|c|c|c|c|c|c|c|c|c|c|c|c|}
\hline$E_{11}$ & $E_{22}$ & $E_{33}$ & $v_{12}$ & $v_{13}$ & $v_{23}$ & $G_{12}$ & $G_{13}$ & $G_{23}$ & $e_{f}$ & $e_{m}$ & $e_{s}$ & $e_{d}$ \\
\hline 1 & 1 & 1 & 1 & 1 & 1 & 1 & 1 & 1 & 0 & 0 & 0 & 0 \\
\hline 0.14 & 0.14 & 0.14 & 0.14 & 0.14 & 0.14 & 0.14 & 0.14 & 0.14 & 1 & 0 & 0 & 0 \\
\hline 1 & 0.2 & 1 & 1 & 1 & 1 & 0.2 & 0.2 & 0.2 & 0 & 1 & 0 & 0 \\
\hline 1 & 1 & 1 & 0 & 0 & 0 & 0 & 1 & 1 & 0 & 0 & 1 & 0 \\
\hline 1 & 1 & 0 & 1 & 0 & 0 & 1 & 0 & 0 & 0 & 0 & 0 & 1 \\
\hline 0.14 & 0.14 & 0.14 & 0.14 & 0.14 & 0.14 & 0.14 & 0.14 & 0.14 & 1 & 1 & 0 & 0 \\
\hline 0.14 & 0.14 & 0.14 & 0 & 0.14 & 0.14 & 0 & 0.14 & 0.14 & 1 & 0 & 1 & 0 \\
\hline 0.14 & 0.14 & 0 & 0.14 & 0 & 0 & 0.14 & 0 & 0 & 1 & 0 & 0 & 1 \\
\hline 1 & 0.2 & 1 & 0 & 1 & 1 & 0 & 0.2 & 0.2 & 0 & 1 & 1 & 0 \\
\hline 1 & 0.2 & 0 & 1 & 0 & 0 & 0.2 & 0 & 0 & 0 & 1 & 0 & 1 \\
\hline 1 & 1 & 0 & 0 & 0 & 0 & 0 & 0 & 0 & 0 & 0 & 1 & 1 \\
\hline 0.14 & 0.14 & 0.14 & 0 & 0.14 & 0.14 & 0 & 0.14 & 0.14 & 1 & 1 & 1 & 0 \\
\hline 0.14 & 0.14 & 0 & 0.14 & 0 & 0 & 0.14 & 0 & 0 & 1 & 1 & 0 & 1 \\
\hline 0.14 & 0.14 & 0 & 0 & 0 & 0 & 0 & 0 & 0 & 1 & 0 & 1 & 1 \\
\hline 1 & 0.2 & 0 & 0 & 0 & 0 & 0 & 0 & 0 & 0 & 1 & 1 & 1 \\
\hline 0.14 & 0.14 & 0 & 0 & 0 & 0 & 0 & 0 & 0 & 1 & 1 & 1 & 1 \\
\hline
\end{tabular}

subroutine USDFLD.

\section{Material Degrade Scheme}

For material degrade, there are two typical degrade modes: abrupt degradation and progressive degradation. In the case of abrupt degradation, i.e. instantaneous degradation, the relative material property reduces to zero or a small value due to the occurrence of damage. Abrupt degradation is widely applied in progressive damage analysis for its easy operation. Unlike abrupt degradation, the material degradation rule is described as a function of damage variables in progressive degradation mode, which can avoid the distortion of local elements due to material abrupt degeneration, particularly in the simulation of dynamic behaviors.

In ABAQUS/Standard, the static problem is usually globally solved to reduce local distortion. Therefore abrupt degradation mode is adopted in the present work. The detail of the material degradation is shown in Table II.

That the damages initiate at the locations of the highest stresses, in the following analysis the locations of highest stresses are evaluated to predict possible locations of damage initiation combined with damage initiation criteria.

\section{NUMERICAL RESULTS}

The material damage initiation criteria and material degradation are implemented by means of ABAQUS user

\section{A. Stress Analysis}

The aim of the present work is to understand the damage modes and failure load of the CFRP laminate with fiber waviness and resin zone, therefore we focus on the stress distribution in composite plies. Based on the expectation
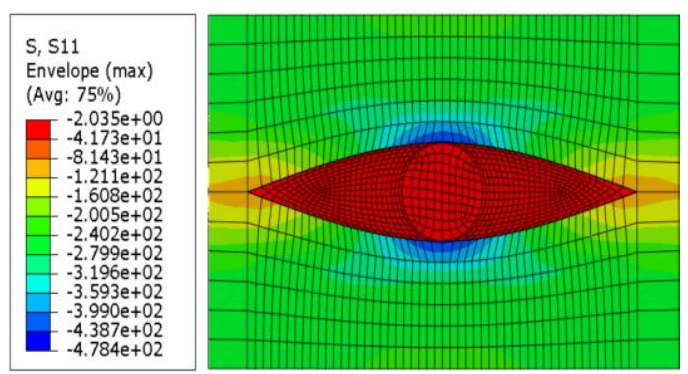

(a) Normal stress in the longitudinal direction $\left(\sigma_{11}\right)$.

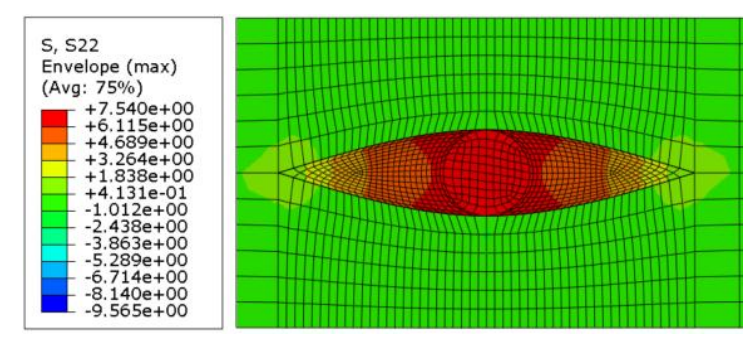

(b) Normal stress in the thickness direction $\left(\sigma_{33}\right)$. 


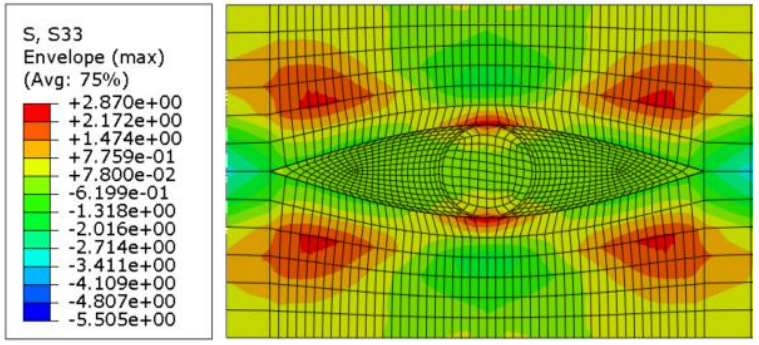

(c) In-plane transverse normal stress $\left(\sigma_{22}\right)$.

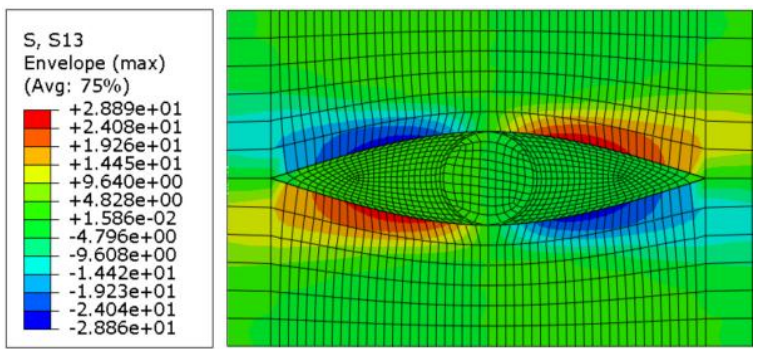

(d) Shear stress in x-z plane $\left(\sigma_{12}\right)$

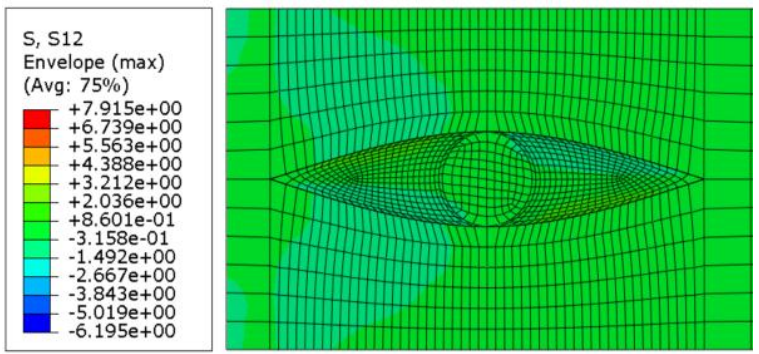

(e) Transverse shear stress in x-y plane $\left(\sigma_{13}\right)$

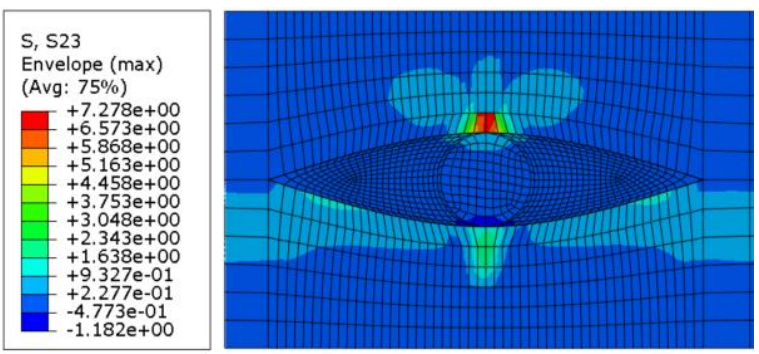

(f) Shear stress in y-z plane $\left(\sigma_{23}\right)$

Fig. 5. Stress distribution at waviness location for $\delta=0.0114 \mathrm{~mm}$.

Stresses distribution at waviness location for compressive displacement $\delta=0.0114 \mathrm{~mm}$ were shown in Fig.5. It has to be pointed out that the $\mathrm{y}$ direction is in accordance with the thickness direction. Accordingly the direction index " 2 " should be exchanged with " 3 " in the stress symbol "Sij" in Fig.5 (b) to (f). In the case of axial compression, the normal stress in the longitudinal direction $\sigma_{11}$ in the composite plies is negative. Thereby the criteria in (2) and (5) would be applied for the onset of fiber failure and fiber-matrix shearing. We can see that in Fig.5, the magnitude of $\sigma_{11}$ is far greater than all the other stress components. The maximum value of $\sigma_{11}$ occurs at the peak of the highest misalignment fiber and it implies the initiation of fiber failure at this location according to (2). High shear stresses $\sigma_{12}, \sigma_{13}$ appear at the location with maximum misalignment angle, which in turn trigger the formation of fiber-matrix shearing here. Tensile stress $\sigma_{33}$ occurs in the resin-zone, although the stress lever is low it may lead to cracks initiation perpendicular to the thickness direction (mode I crack initiation) for the low fracture resistant of the resin, which is consistent with the results in [10].

\section{B. Failure Modes in the Model}

For a profound understanding of damage initiation and progression, damage modes are discussed in the following section.

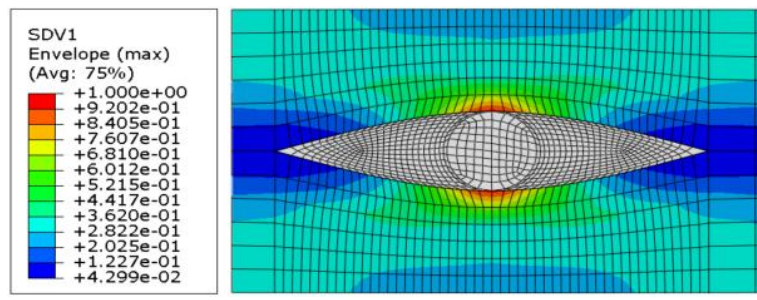

(a) fiber failure initiation at $\delta=0.0222 \mathrm{~mm}$

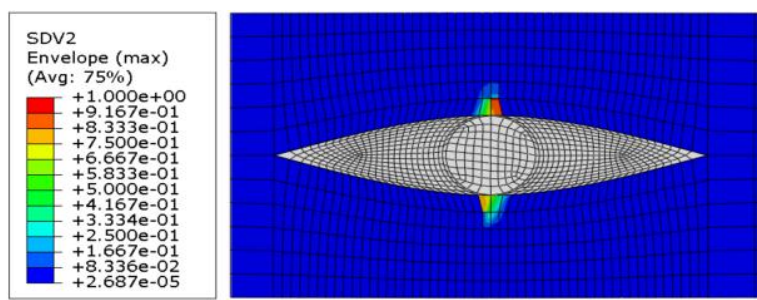

(b) Matrix failure initiation at $\delta=0.024 \mathrm{~mm}$.

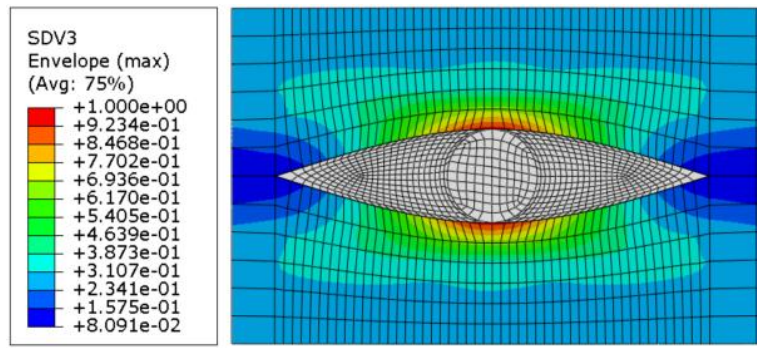

(c) fiber-matrix shearing initiation $\delta=0.024 \mathrm{~mm}$

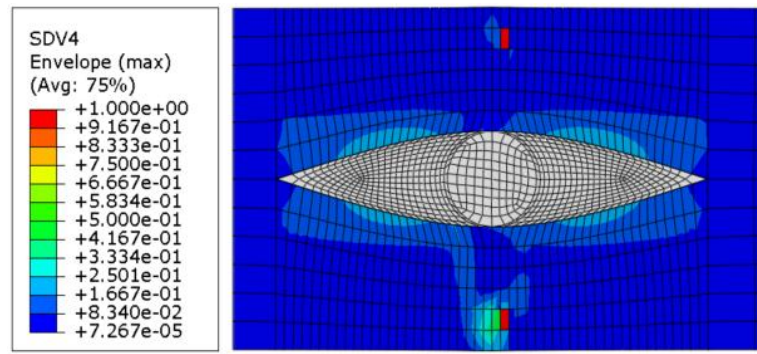

(d) Delamination initiation at $\delta=0.0294 \mathrm{~mm}$

Fig. 6. Damage initiation at waviness location

To capture the sequence of different damage modes, the locations and moments of initiation of four damages are shown in Fig.6. As can be seen, fiber failure occurs first among all the damage modes, at the top and bottom of the resin zone when $\delta=0.0222 \mathrm{~mm}$. This is followed by matrix failure and fiber-matrix shearing, then shortly afterwards by delamination. It can be inferred from Fig. 6 that the damage ranges caused by fiber failure and fiber-matrix shearing are larger than those of matrix failure and delamination.

To confirm that the leading damage mechanism is one of the four damage mode, the model was run without a certain failure mode. For comparison, the model of a UD laminate specimen without any defect was built to perform the identical analysis. The corresponding load vs. displacement curves were shown in Fig.7.

It is shown in Fig.7(a) that the curve of reaction load vs. 
displacement not incorporating fiber failure in the model obviously differs from that with four damage modes, while none of the other three failure modes has any influence on the failure load of the laminate. There is a knockdown in the curve of the reaction load vs. displacement for the laminate without any defect, although all damage modes were considered in the model corresponding model. It is noted that the defect drastically reduces the level of failure load of the laminate, with a reduction of the pristine strength to about $60 \%$.

However, it should be noted that there is no knockdown in the curve without fiber failure for the compressive displacement $\delta<0.06 \mathrm{~mm}$. Is this related to the waviness severity? In order to be clear on this point, the curve of reaction load vs. displacement for the height of the resin-zone $\mathrm{H}=0.2 \mathrm{~mm}$ is shown in Fig.7(b). Similar result is found, which infers that the waviness severity has no influence on the damage mode in the present model. Although the present FE model may be improved to deal with this issue, it is clearly indicated that fiber failure is the dominant mechanism for the laminate with both local waviness and a resin zone.

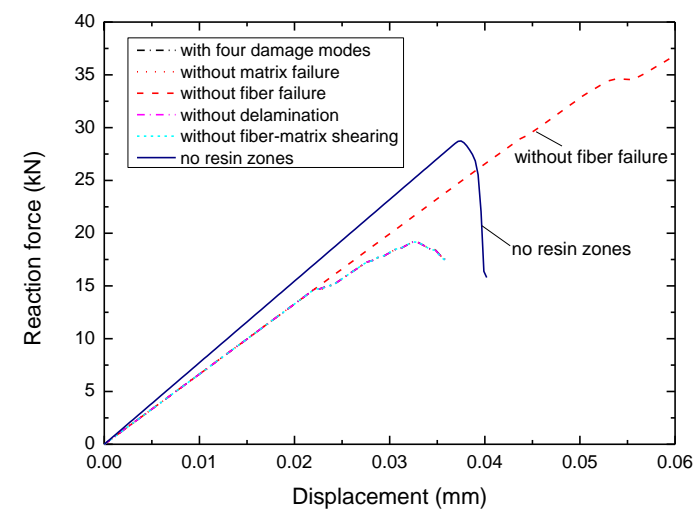

(a)

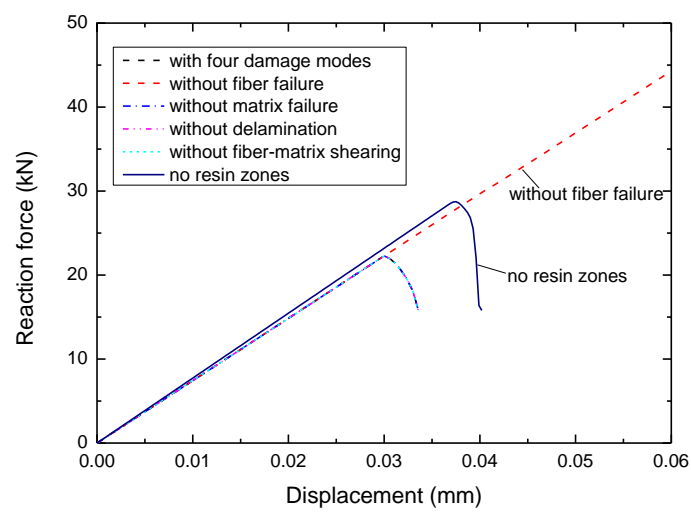

(b)

Fig.7. Predicted load vs. displacement curves for the case of a single resin-zone: (a) the height of the resin-zone is $0.5 \mathrm{~mm}$; (b) the height of the resin-zone is $0.2 \mathrm{~mm}$.

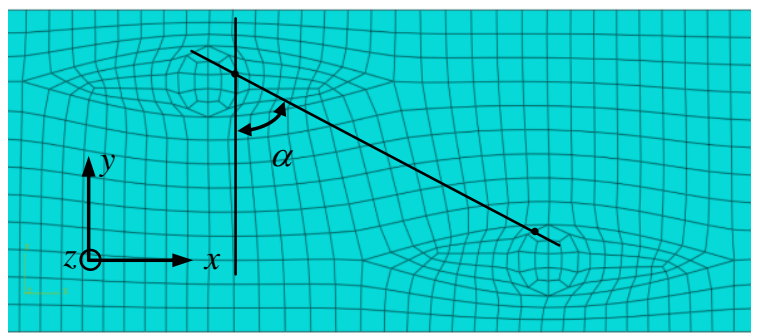

Fig. 8. Defect geometry and FE mesh for the case with two resin-zones
To illustrate the interaction between resin-zones, a similar FE progressive damage model with two resin-zones in the laminate was built up in ABAQUS/Standard, as shown in Fig.8. The only difference between the previous model in Fig. 4 and the present model here is only the number of resin-zones. The configuration of the defect in Fig. 8 can be described by the angle $\alpha$. Example plots of the stress distributions are presented in Fig. 9.

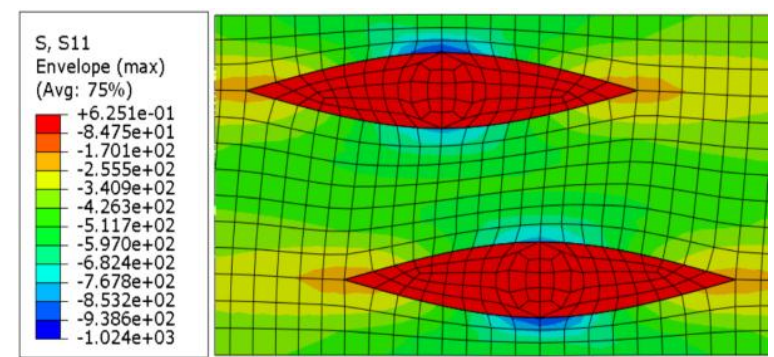

(a) Normal stress in the longitudinal direction $\left(\sigma_{11}\right)$.

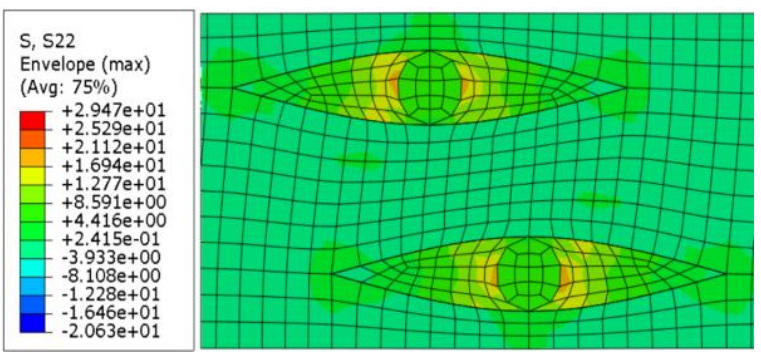

(b) Normal stress in the thickness direction $\left(\sigma_{33}\right)$.

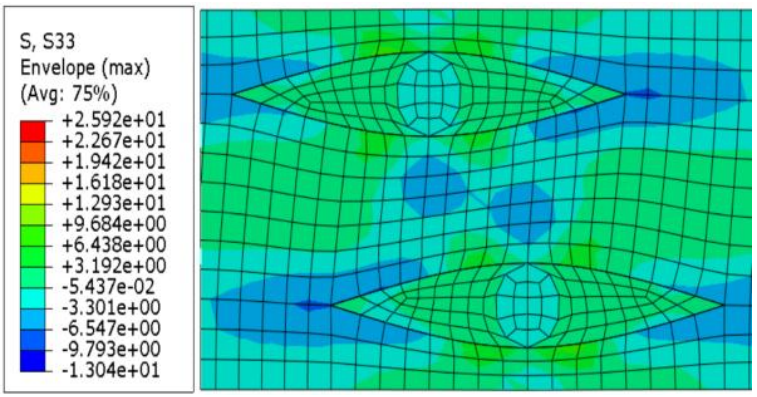

(c) In-plane transverse normal stress $\left(\sigma_{22}\right)$.

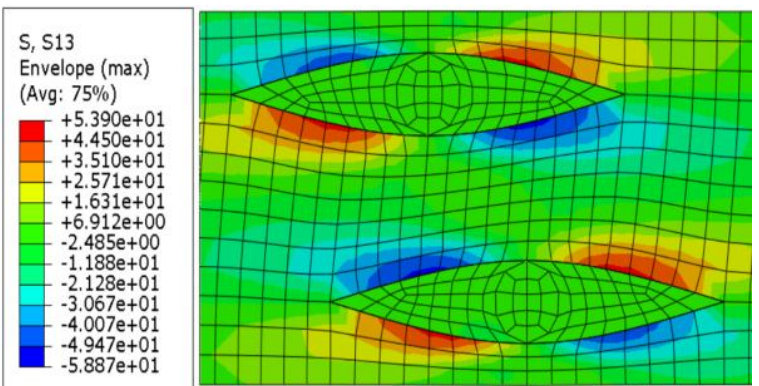

(d) Shear stress in x-z plane $\left(\sigma_{12}\right)$.

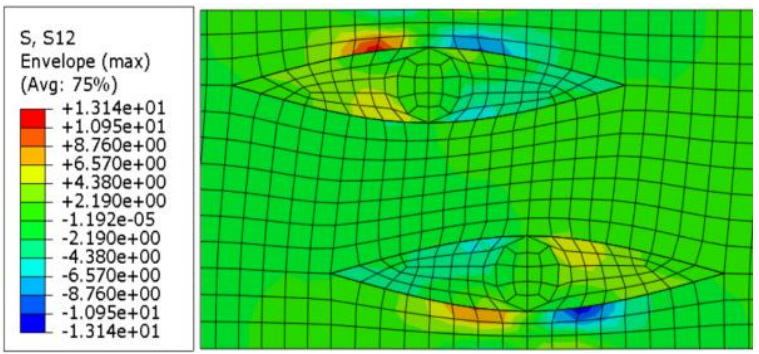

(e) Transverse shear stress in $x-y$ plane $\left(\sigma_{13}\right)$. 


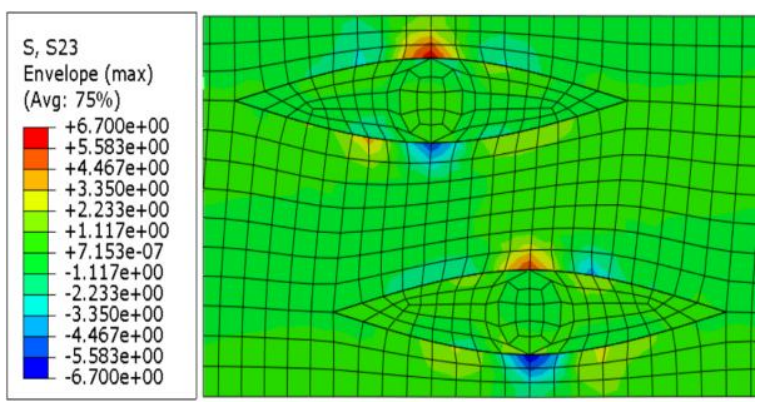

(f) Shear stress in y-z plane $\left(\sigma_{23}\right)$

Fig. 9. Stress distribution at waviness locationwith two resin-zones for $\delta=0.0216 \mathrm{~mm}\left(\alpha=30^{\circ}\right)$.

Similar to the case of a single resin-zone, the longitudinal normal stress in the composite plies is negative with high stress level. The locations of higher shear stresses $\sigma_{12}, \sigma_{13}$ coincide to the maximum wrinkle angles as the previous case. It should be noted that, for the case of two resin-zones, once damage initiates in the area with high stress level between two resin-zones, the failure region would extend gradually as damage propagation until connecting into a large damage band finally. This is the interaction mechanism of two resin-zones, which is different from the case of a single resin zone.

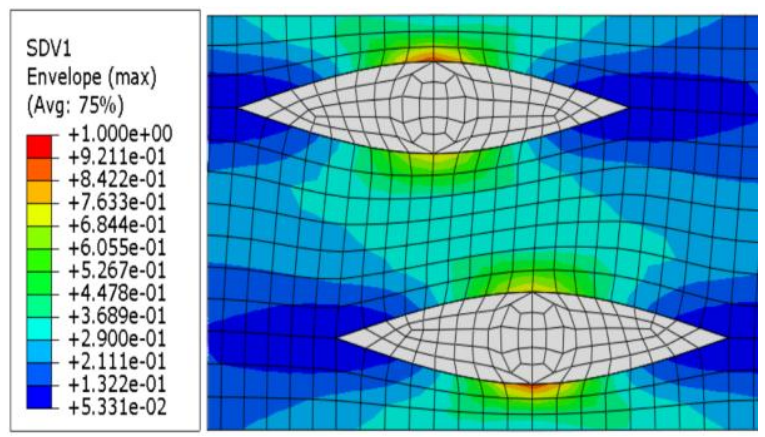

(a) Fiber failure.

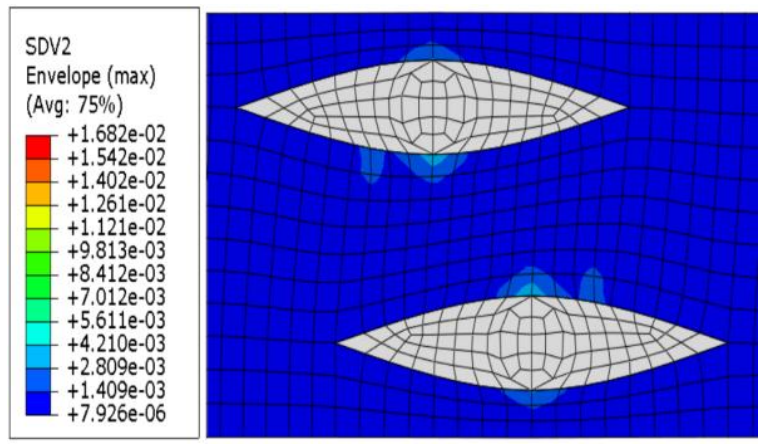

(b) matrix failure.

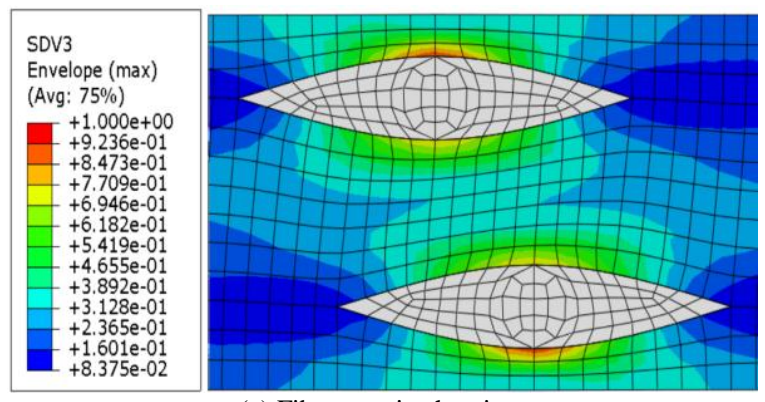

(c) Fiber-matrix shearing.

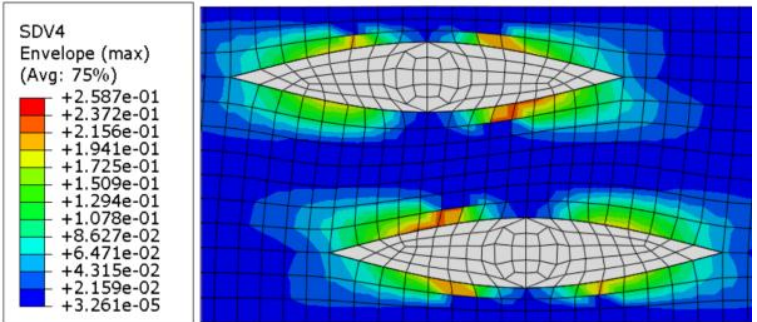

(d) Delamination

Fig. 10. Patterns of damage at maximum load (For $\alpha=30^{\circ}$ ).

Fig. 10(a)-(d) show four damage patterns of the laminate with two resin-zones at maximum load. It could be inferred that fiber failure and fiber-matrix shearing occur at the first moment, then followed by interlaminar delamination and finally matrix failure. The observed damage patterns of a laminate with two resin-zones were presented experimentally in [10], as shown in Fig.11 (a) for $\alpha=35^{\circ}$. It can be seen clearly that the locations of fiber fracture and delamination predicted in the present model (see in Fig.10) are consistent with those in Fig. 11 (a).

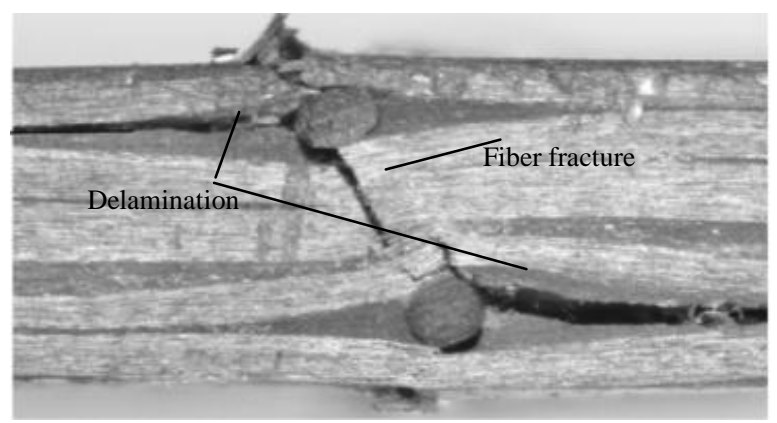

(a) Fracture pattern.

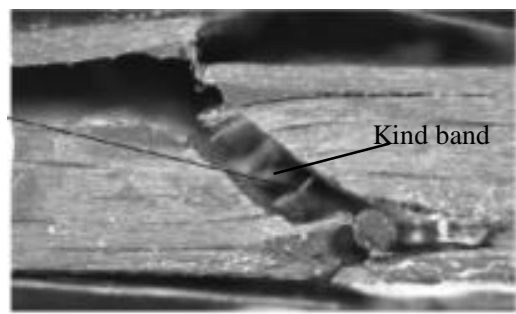

(b) Final collapse.

Fig. 11. Experimental photoes of fracture patterns under axial compression in [10].

The final collapse pattern of the case of two resin zones was shown in Fig. 11(b), with a kind band at the junction of the two resin zones. A kind band is induced by fiber breakage and fiber micro-buckling due to matrix plastic yield [11], [12].

As before, to better demonstrate the significance of different damage modes, the model was run with and without fiber failure or delamination mode. The results are shown in Fig. 12. The effect of fiber failure is similar to the case of a single resin zone. However, it is worthy of noting that inter-ply delamination also plays a significant role on the failure load, which is different from the case of a single resin zone. 


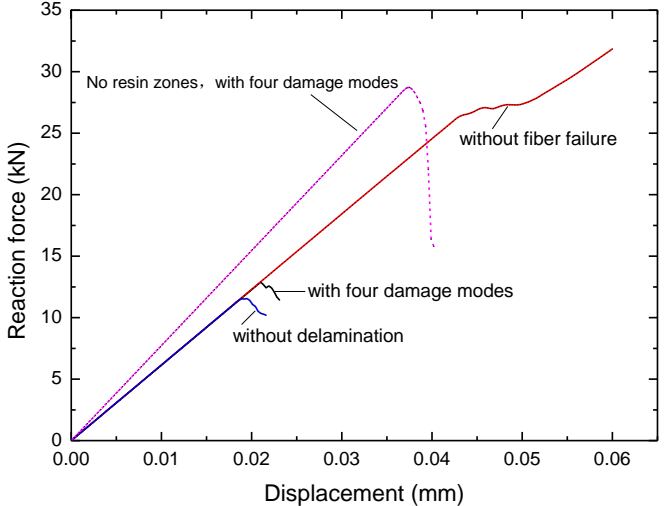

Fig. 12. Predicted load vs. displacement curves for the case of two resin-zones.

\section{Influence of Defect Configuration on Failure Load}

The following section is to evaluate the effect of defect geometry on the compressive strength of the laminate by comparison with the compressive strength of laminate without any defect.

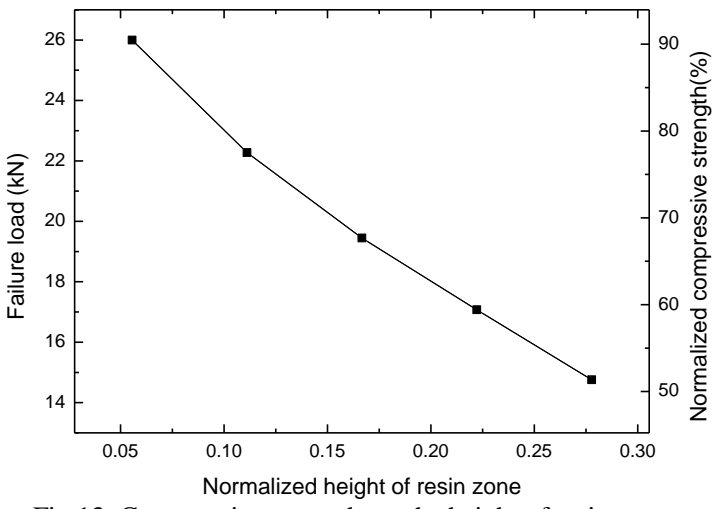

Fig.13. Compressive strength vs. the height of resin-zone.

For the case of a single resin-zone, the dependence of the compressive strength on the height of the resin-zone normalized by the thickness of the laminate is shown in Fig. 13 , in which the width of the resin-zone keeps constant $(2.4 \mathrm{~mm}, 40 \%$ of the laminate). The right $\mathrm{Y}$ is the compressive strength normalized by the strength of no-defect laminate. It can be seen that the failure load has a nearly linear decrease with the increase of the normalized height. For the normalized height is 0.28 , the strength reduces to $52.4 \%$ of the pristine strength.

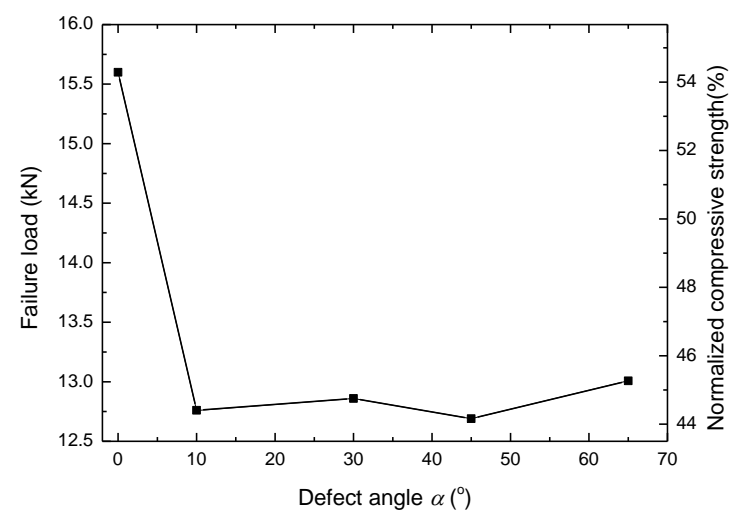

Fig. 14. Compressive strength vs. defect angle.

The influence of the defect angle on the compressive strength is illustrated in Fig.14. For $\alpha=0^{\circ}$, the laminate has the highest level of failure load, with a reduction of the strength to about $54 \%$ of the pristine strength without any defect. With the increase of the defect angle, the compressive strength drops down with a minimum value for a defect angle $\alpha=45^{\circ}$. This is in good agreement with the result in [10].

\section{CONCLUSION}

Based on the proposed progressive damage model, the failure behavior of a CFRP laminate with both fiber waviness and resin zones was described quantitatively. The results show that for the laminate with both waviness and resin zones, the dominant damage mechanism is fiber failure, which is different from the results given by the literature involved only fiber waviness. As described in the literature [11], [12],matrix plasticity plays an important role on the compression failure modes of axially loaded UD CFRP laminate. However, the damage model proposed in the present work did not consider the plastic effect, particularly in the waviness location. Therefore, in order to illustrate the exact damage behavior of the CFRP with both waviness and resin-zones, a combined elastoplastic progressive damage model would be developed in the future work.

\section{ACKNOWLEDGMENT}

The authors thank Jing-fen Chen for her technical guidance in $\mathrm{FE}$ simulation.

\section{REFERENCES}

[1] J. Wang, K. D. Potter, K. Hazra, and M. R. Wisnom, "Experimental Fabrication and characterization of out-of-plane fibre waviness in continuous fibre-reinforced composites," Journal of Composite Materials, vol. 46, no.17, pp. 2041-2053, Aug 2012.

[2] L. D. Bloom, J. Wang, and K. D. Potter, "Damage progression and defect sensitivity: An experimental study of representative wrinkles in tension," Composite Part B: Engineering, vol. 45, no.1, pp. 449-458, Feb 2013.

[3] K. Potter, B. Khan, M. Wisnom, T. Bell, and J Stevens, "Variability, fibre waviness and misalignment in the determination of the properties of composite materials and structures," Composites: Part A: Applied Science Manufacturing, vol. 39, pp.1343-1354, Sept 2008.

[4] R F. ElHajjar amd S. S. Shams, "Compression testing of continuous fiber reinforced polymer composites with out-of-plane fiber waviness and circular notches," Polymer Testing, vol.35, pp. 45-55, May 2014.

[5] H. M. Hsiao and I. M. Daniel, "Effect of fiber waviness on stiffness and strength reduction of unidirectional composites under compressive loading," Composites Science and Technology, vol. 56 no. 5, pp. 581-593, May 1996.

[6] H.M. Hsiao and I.M. Daniel, "Elastic properties of composites with fiber waviness," Compos Part A: Applied Science Manufacturing, vol.27, no. 10, pp. 931-941, Oct 1996

[7] H. J. Chun, J. Y. Shin and I. M. Daniel, "Effect of material and geometric nonlinearities on the tensile and compressive behavior of composite materials with fiber waviness," Composites Science and Technology, vol. 61, no. 1, pp. 125-134, Jan 2011.

[8] S. Mukhopadhyay, M. I. Jones, and S. R. Hallett, "Compressive failure of laminates containing an embedded wrinkle: experimental and numerical study," Compos Part A: Applied Science Manufacturing, vol. 73, pp.132-142, June 2015.

[9] N. Xie, R. A. Smith, S. Mukhopadhyay, and S. R. Hallett, "A numerical strudy on the influence of composite winkle defect geometry on compressive strength," Materials and Design, vol. 140, pp. 7-20, Feb 2018

[10] S. Hörrmann, A. Adumitroaie, C. Viechtbauer, and M. Schagerl, "The effect of fiber waviness on the fatigue life of CFRP materials," International Journal of Fatigue, vol. 90, pp. 139-147, Sept 2016. 
[11] A. P. Mouritz and B. N. Cox, "A mechanistic approach to the properties of stitched laminates," Compos Part A: Applied Science Manufacturing, vol. 31, no. 1, pp.1-27, Jan 2000

[12] N. Fleck. "Compressive failure of fiber composites," in Advances in Applied Mechanics, J. W. Hutchinson, T. Y. Wu, Ed. San Diego: Academic press Inc., 1997, vol. 33, pp. 43-119.

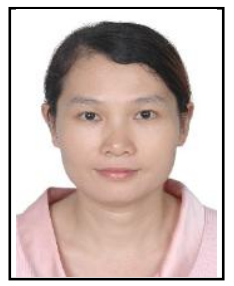

Z. Ning was born in Guangxi province, China, Oct 1976. Educational background is as following.

She received her Ph.D in engineering mechanics, Jinan University, Guangzhou, China, 2011.

She received her M.S in solid mechanics, Hunan University, Changsha, China, 2002.

She received her B.S in engineering mechanics, Hunan University, Changsha, China, 1999.

The major fields of study are micromechanics on composites and nonlinearity analysis of Structures.

She is currently an associate professor in School of Mechanics and Construction Engineering, Jinan University, China. She had worked as a visiting scholar at the Engineering Mechanics and Composites Research Laboratory, University of Wisconsin, U.S.A., from Feb.2015 to Feb.2016. Her recent publications are listed as follows:

Z. Ning, R. Liu, R. F. Elhajjar, and F. Wang, "Micro-modeling of thermal properties in carbon fibers reinforced polymer composites with fiber breaks or delamination," Composites Part B:Engineering, vol. 114, pp. 247-255, Apr 2017.

Z. Ning, R. Liu, and H. Liu, "Micro-modeling of thermal resistance change in fibers reinforced composites with fiber breakage," Acta Materiae Compositae Sinica, vol. 34, no.1, pp. 112-120, Jan 2017.

Her research interests include multiscale analysis on composite materials, NDT in FRP composites.

Dr. Ning is the member of the council of Society of Theoretical and Applied Mechanics, Guangdong Province, China.

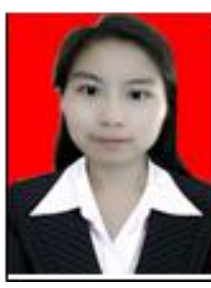

C. Xu was born in Shandong province, China, Oct 1987. Educational background is as following.

M.S in Engineering mechanics, Jinan University, Guangzhou, China, 2018.

She received her B.S in Engineering mechanics, Taiyuan University of Science and Technology, Taiyuan, China, 2016

The major field of study is numerical simulation of FRP composites with embedded defects. She is currently a technician in an automobile company in Shenzhen, China. Her research interests include multiscale analysis on composite materials, NDT in FRP composites.

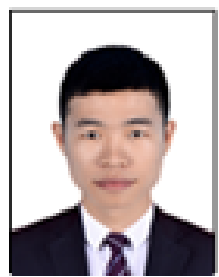

G. Huo was born in Guangdong province, China, Apr 1994. He got his B.S in water resources and hydro-electric engineering, Xian University of Technology, Xian, China, 2016. He is studying in Jinan University for his master degree in engineering mechanics. The major field of study is solid mechanics.

His research interests include NDE in FRP composites and deep learning application in prediction of properties of FRP composites. 